\title{
Effect of Teriparatide on Subsequent Vertebral Fractures after Instrumented Fusion Surgery for Osteoporotic Vertebral Fractures with Neurological Deficits
}

Keishi Maruo, Toshiya Tachibana, Fumihiro Arizumi, Kazuki Kusuyama, Kazuya Kishima, Shinichi Yoshiya

Department of Orthopaedic Surgery, Hyogo College of Medicine, Nishinomiya, Japan

Study Design: Retrospective case review.

Purpose: To assess the incidence and effect of teriparatide (TP) on subsequent vertebral fractures following a long-instrumented fusion surgery for osteoporotic vertebral fractures (OVFs).

Overview of Literature: TP treatment may be a useful strategy for patients with OVFs treated with a long-instrumented surgery.

Methods: Overall, 47 patients who underwent long-instrumented fusion surgery ( $\geq 3$ levels) for OVFs with neurological deficits between 2010 and 2013 were enrolled. The mean age of the subjects was 76 years; the study population comprised 20 males and 27 females. The mean follow-up duration was 23 months. The average of fused vertebrae was 4.9. TP was used for 19 patients who comprised the TP group. The incidence of subsequent VFs was estimated with Kaplan-Meier analyses and compared between the TP and non-TP groups using the log-rank test. Risk factors were evaluated using a Cox proportional hazards model.

Results: A total of $38 \%$ (18/47 cases) of the subjects were identified with subsequent VFs. There were no significant differences in the age, sex, fused levels, presence of prevalent fractures, and correction loss of the two groups. The occurrence of subsequent VFs was lower in the TP group than in the non-TP group (16\% vs. 54\%, $p=0.014)$. The log-rank test revealed that the TP treatment significantly reduced the risk of subsequent VFs $(p=0.048)$. A Cox proportional hazards model revealed that preoperative TP treatment is only a protective factor of subsequent VFs after instrumented fusion surgery for OVFs (hazard ratio, $0.281 ; p=0.047$ ).

Conclusions: In this retrospective study, pre- and postoperative TP treatment significantly reduced the incidence of subsequent VFs after instrumented fusion surgery for OVFs. A prospective randomized study is warranted to determine the efficacy of TP treatments.

Keywords: Osteoporosis; Vertebral fractures; Teriparatide; Spinal surgery

\section{Introduction}

Osteoporotic vertebral fractures (OVFs) are associated with a compromised quality of life, morbidity, and mortality [1]. Majority of OVF cases can be conservatively treated with bed rest, bracing, osteoporosis treatment, and pain management. However, vertebroplasty (VP) or balloon kyphoplasty (BKP) have been reported as effective treatments for persistent painful OVFs [2-5]. Some cases progress vertebral nonunion and neurological complica-

Received Apr 24, 2018; Revised Jul 25, 2018; Accepted Aug 22, 2018

Corresponding author: Keishi Maruo

Department of Orthopaedic Surgery, Hyogo College of Medicine, Mukogawa-cho, Nishinomiya, Hyogo 663-8501, Japan

Tel: +81-798-45-6452, Fax: +81-798-45-6453, E-mail: kmaruo@hyo-med.ac.jp 
tions with middle column compromise [6]. However, in case of severe vertebral collapse with neurological deficits, it is difficult to maintain neurological function and stabilize the fracture using VP or BKP. In general, VP and BKP are contraindicated in patients with posterior wall damage owing to possible neural complications caused by epidural cement leakage. Few studies have confirmed the efficacy of spinal reconstruction surgeries, such as anterior spinal fusion (ASF), posterior spinal fusion (PSF), pedicle subtraction osteotomy (PSO), and anterior and posterior spinal fusion (AP), with or without VP in these conditions [7-13]. Recently, VP and BKP have been associated with an increased risk of subsequent VFs [14,15]. Therefore, long-instrumented fusion surgery for OVFs may increase the risk of subsequent VFs. However, few studies have reported incidences of subsequent VFs after instrumented fusion surgery for OVFs. Several studies have shown that teriparatide (TP) decreases the risk of subsequent VFs after VP or BKP [16,17]. Our hypothesis was as follows: (1) long-instrumented fusion surgery for OVFs with neurological deficits increases the risk of subsequent VFs and (2) TP decreases the risk of subsequent VFs. This study aimed to assess the incidence and effect of TP on subsequent VFs after long-instrumented fusion surgery for OVFs.

\section{Materials and Methods}

\section{Patient population}

Total 47 patients who underwent long-instrumented fusion surgery ( $\geq 3$ levels) for OVFs from 2011 to 2015 were enrolled. Patients with tumors, pyogenic vertebral osteomyelitis, those who had undergone only VP or BKP, those who were followed-up for $<1$ year, and those with a history of previous spinal fusion surgery were excluded. The mean age at surgery was $76 \pm 6.3$ years (range, $63-88$ years); the study population included 20 males and 27 females. The mean follow-up period was $23 \pm 14$ months (range, 12-59 months). The patients were regularly followed-up every 3 months postoperatively. Approval was obtained from the Institutional Review Board of Hyogo College of Medicine for patient enrollment and data collection protocols (IRB approval no., 2186). Majority of the cases involved the thoracolumbar region (T12: 42\%, 20 cases and L1: 21\%, 10 cases) (Fig. 1). Of the 47 patients, 15 had prevalent VF, and eight had diffuse idiopathic skeletal hyperostosis. Only five patients had taken bisphospho-

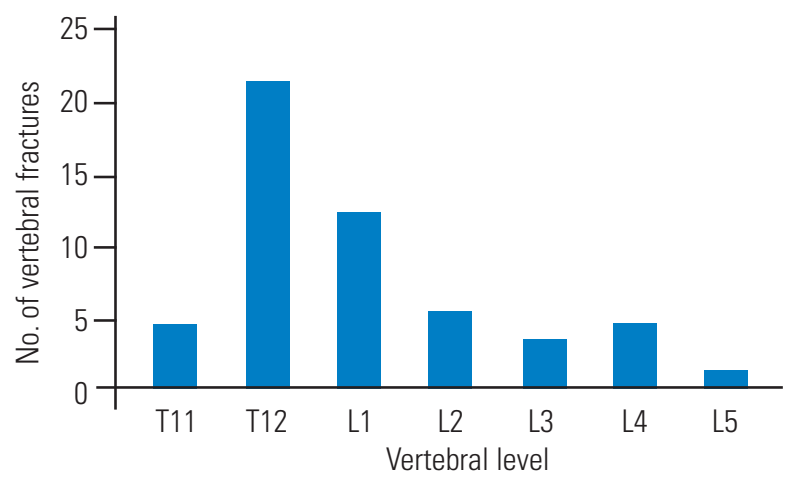

Fig. 1. Distribution of the initial vertebral fracture.

nates, and three patients received vitamin $\mathrm{D}$ and calcium supplementation for osteoporosis at the time of OVFs diagnosis. All the patients were prescribed bisphosphonate or TP preoperatively. Majority of the patients had insufficient bone union after conservative treatment including lumbar orthosis and bed rest after OVFs. Indications for the use of TP treatment were prevalent multilevel VFs, severe vertebral collapse $(>50 \%)$, and the presence of marked intravertebral vacuum cleft, especially since 2011. TP treatment was initiated for 19 patients who received a subcutaneous injection $(20 \mu \mathrm{g})$ once daily at the time of diagnosis of OVFs with neurological deficits. The median duration of previous treatment with antiresorptive agents and TP was $1.3 \pm 0.3$ months and $1.5 \pm 0.4$ months, respectively. Bisphosphonates or TP are contentiously administered postoperatively.

\section{Surgical management}

All the patients were initially treated conservatively with bed rest and thoracolumbar orthosis for several months. Surgical treatments were indicated for patients with progressive neurological deficits caused by severe vertebral collapse or spine instability [18]. All patients, except two, had neurological deficits. These two patients were treated surgically for persistent severe back pain due to vertebral pseudarthrosis. We mainly selected VP with PSF in case of severe vertebral collapse with intravertebral vacuum cleft. VP was performed using calcium phosphate cement (Biopex; Pentax Co., Tokyo, Japan) or hydroxyapatite blocks (Pentax Co.), as per the surgeon's preference. ASF and PSF or osteotomy was indicated for severe biconcave vertebral fracture without intravertebral vacuum cleft. Types of surgery included VP with PSF in 36 cases, AP in three cases, PSF without VP in two cases, posterior lumbar interbody 
fusion in two cases, and osteotomy and PSF in four cases. The average number of fused vertebrae was 4.9 (range, 3-7 vertebrae). Subsequent VF was defined as a reduction of $\geq 20 \%$ in the anterior, middle, or posterior vertebral height at adjacent levels and distant levels.

\section{Clinical assessment}

The occurrence of subsequent postoperative VFs, complications, reoperation, and Visual Analog Scale scores for low back pain (LBP) was reviewed to evaluate the clinical outcomes. Activities of daily living were assessed using the Barthel index. The LBP score was evaluated using the Japanese Orthopaedic Association score (0, none; 1, occasional and slight; 2, occasional and moderate to severe; 3 , continuous and severe). Radiographic measurements included the assessment of the kyphotic angle (KA) between the cranial endplate of upper instrumented vertebra (UIV) and the caudal endplate of the lower instrumented vertebra (LIV) on lateral plain radiography. Age, sex, fused levels, KA, fusion rate, correction loss, subsequent VFs, and LBP score were compared between the TP and nonTP groups.

\section{Statistical analyses}

Continuous variables are presented as means \pm standard deviations with ranges, and categorical variables are presented in terms of frequencies and percentages. The independent Student $t$-test was used to compare the two groups. The chi-square test or the Fisher's exact test was used, as required, if a frequency of any cell in a contingency table was $<5$. All the $p$-values are two-sided, and $p$ values $<0.05$ were considered to indicate statistical significance. The occurrence of subsequent VFs was estimated using Kaplan-Meier analyses and compared between the groups using the log-rank test. The risk factors were evaluated using a Cox proportional hazards model. SPSS ver. 17.0 (SPSS Inc., Chicago, IL, USA) was used for performing the statistical analyses.

\section{Results}

Total 38\% (18/47 cases, 30 vertebrae) were identified in the subsequent VFs. Of the 18 patients, 10 had 1 level VF, five had 2 level VFs, two patients had 3 level VFs, and one patient had 4 level VFs (Table 1). The median time of VFs
Table 1. Clinical data of subsequent vertebral fractures

\begin{tabular}{lc}
$\begin{array}{l}\text { Variable } \\
\text { Total no. of cases }\end{array}$ & Value \\
\hline $\begin{array}{l}\text { No. of cases with subsequent vertebral } \\
\text { fracture }\end{array}$ & 18 (38) (30 vertebrae) \\
\hline $\begin{array}{l}\text { No. of fracture } \\
1 \text { Level }\end{array}$ & 10 \\
\hline 2 Levels & 5 \\
\hline 3 Levels & 2 \\
\hline 4 Levels & 1 \\
\hline Timing of subsequent fracture & 4 mo \\
\hline Median & 8 mo (1-45 mo) \\
\hline Mean (range) & $7(39)$ \\
\hline$<3$ mo & $6(33)$ \\
\hline$<6$ mo & $2(11)$ \\
\hline$<1$ yr & $3(17)$ \\
\hline$>1$ yr & 8 \\
\hline No. of patients with symptomatic fracture & \\
\hline
\end{tabular}

Values are presented as number (\%), unless otherwise stated.

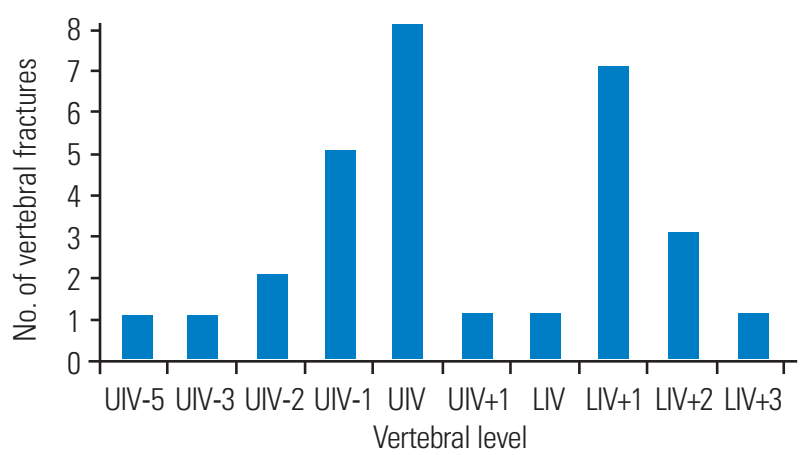

Fig. 2. Distribution of subsequent vertebral fractures. UIV, upper instrumented vertebra; LIV, lower instrumented vertebra.

from the index surgery was 4 months. Of the 18 patients, seven (39\%) had subsequent VFs within 3 months, six (33\%) within 3-6 months, two (11\%) within 6 months to 1 year, and three (17\%) more than 1 year after the index surgery. Eight patients had symptomatic VFs, and 10 patients had subsequent asymptomatic VFs at the regular follow-up. Fracture at the UIV was the most common subsequent VF. LIV +1 was the second most common subsequent VF (Fig. 2). All the subsequent VFs were treated conservatively with braces and pain medication. The overall occurrence of subsequent VFs was $21 \%$ at 1 year and $32 \%$ at 2 years according to the Kaplan-Meier estimate (Fig. 3). Total six patients required revision surgery because of complications, including surgical site 


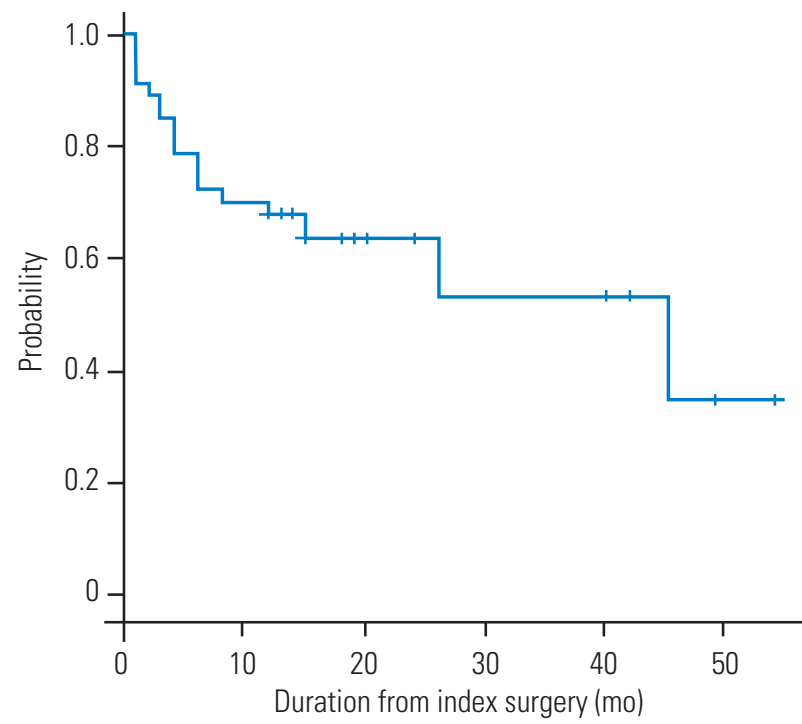

Fig. 3. A Kaplan-Meier survival curve was constructed for estimating the subsequent vertebral fractures after surgery. This curve shows the probability of survival without subsequent fracture over.

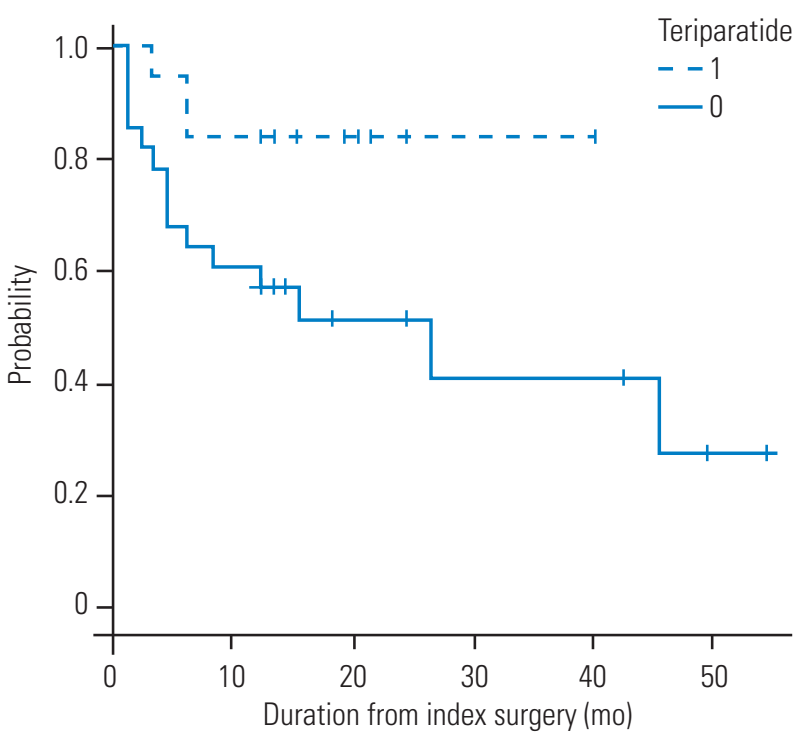

Fig. 4. A Kaplan-Meier survival curve for the teriparatide treatment group (dotted line) vs. the non-teriparatide treatment group (solid line). The curve reveals a high incidence of subsequent fracture that was observed in the non-teriparatide treatment group.

Table 2. Comparison of variables between the non-TP group and the TP group

\begin{tabular}{lccc} 
Variable & Non-TP group $(\mathrm{n}=28)$ & TP group $(\mathrm{n}=19)$ & $p$-value \\
\hline Age (yr) & $75.5 \pm 6.9$ & $75.6 \pm 5.7$ & 0.931 \\
Sex (female) & $78(21)$ & $68(13)$ & 0.786 \\
\hline Fused levels & $4.9^{\circ} 1.0$ & $5.0 \pm 1.2$ & 0.116 \\
\hline Postoperative KA (immediate) & $12.1^{\circ} \pm 7.3^{\circ}$ & $14.2^{\circ} \pm 14.0^{\circ}$ & 0.555 \\
\hline Postoperative KA (final follow-up) & $21.4^{\circ} \pm 10.1^{\circ}$ & $20.5^{\circ} \pm 12.3^{\circ}$ & 0.801 \\
Loss of correction & $9.3^{\circ} \pm 7.8^{\circ}$ & $6.3^{\circ} \pm 7.8^{\circ}$ & 0.278 \\
Subsequent fractures & $54(15)$ & $16(3)$ & $0.014^{\mathrm{a})}$ \\
Low back pain score & $1.9_{ \pm 0.5}$ & $2.1^{\circ} 0.7$ & 0.244 \\
\hline
\end{tabular}

Values are presented as mean \pm standard deviation or \% (number).

TP, teriparatide; KA, kyphotic angle.

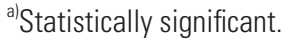

infection (four cases) and instrumentation failure (two cases). One patient required extension to distal level due to distal junctional kyphosis, and another patient required extension to cranial level because of the screw back out. Subsequent VFs did not relate to revision surgery. The fusion rate was similar between the TP group and the nonTP group ( $89 \%$ versus $95 \%, p=0.89$ ). The mean Barthel index significantly increased from $55.1 \pm 11.7$ to $80.2 \pm 12.2$ $(p<0.001)$ at the last follow-up. Preoperative KA between the UIV and the LIV was $24.5^{\circ} \pm 15.0^{\circ}$. The KA immediately after the surgery was $13.0^{\circ} \pm 10.2^{\circ}$. Postoperative KA at the final follow-up was $21.2^{\circ} \pm 10.2^{\circ}$. The mean loss of correction was $8.1^{\circ} \pm 10.2^{\circ}$. There were no significant dif- ferences in the age, sex, fused levels, presence of prevalent fracture, postoperative KA (immediately and at the final follow-up), and LBP score between the TP group and the non-TP group (Table 2). The occurrence of subsequent vertebral fracture was lower in the TP group (16\% versus $54 \%, p=0.014)$. The log-rank test revealed that the TP treatment significantly reduced the risk of subsequent VFs ( $p=0.048$ ) (Fig. 4). A Cox proportional hazards model revealed that the preoperative TP treatment was only a protective factor for subsequent VFs after instrumented fusion surgery for osteoporotic vertebral collapse (hazard ratio, $0.281 ; 95 \%$ confidence interval, $0.08-0.985$; $p=0.047$ ). 


\section{Discussion}

In our study, total $38 \%$ ( $18 / 47$ cases, 30 vertebrae) subjects were identified with subsequent VFs after instrumentation surgery for OVFs with neurological deficits. The incidence of subsequent VFs after instrumentation surgery, including PSF, PSF+VP, PSO, and ASF was reported to range from $14 \%$ to $55 \%$ [7,8,10-12]. Ataka et al. [7] reported that subsequent VFs were found in $50 \%$ (7/14 cases) of the subjects after posterior long-instrumented fusion surgery. Nakashima et al. [10] reported subsequent VFs in 33\% (15/45) of the subjects after AP (24 cases) and VP+PSF (21 cases) surgery. Okuda et al. [11] reported that subsequent VF were found in $40 \%$ (16/40 cases) after surgery (ASF $15 \%$, PSO $86 \%$ ). Kashii et al. [8] reported a rate of $55 \%$ for subsequent VFs after surgery (VP+PSF, ASF and PSO). It remains controversial whether the vertebral augmentation procedure increased the risk of subsequent VFs or it was the result of the natural history of osteoporosis. Several studies have demonstrated a higher rate of subsequent VFs after kyphoplasty compared with the data from natural history for untreated fractures $[14,15]$. In contrast, the vertebral augmentation procedure does not increase the incidence of new vertebral fractures $[2,19,20]$. Recently, a meta-analysis of randomized controlled trials showed no significant differences between the incidences of subsequent VFs after conservative and vertebral augmentation treatments $[21,22]$. The present results indicate that the incidences of subsequent VFs were similar to previously described incidences.

Various surgical procedures have been reported for the treatment of OVFs with neurological deficit. Further, the efficacy of spinal reconstruction techniques, such as ASF, PSF+VP, PSF alone, PSO, and AP has also been reported [7-13]. Concurrently, a high complication rate was identified in terms of correction loss, screw loosening, subsequent VFs, and instrumentation failure after instrumentation surgery in fragile osteoporotic bones. Katsumi et al. [9] reported that the efficacy and safety of $\mathrm{VP}+\mathrm{PSF}$ without neural decompression and the mean correction loss of the KA in the fused area at the 2-year follow-up was $13.6^{\circ} \pm 6.0^{\circ}$. They recommended short-segment VP+PSF for elderly patients. Nakashima et al. [10] demonstrated that correction loss was significantly greater in the VP+PSF group $\left(7.0^{\circ} \pm 6.0^{\circ}\right)$ compared to that in the AP group $\left(13.4^{\circ} \pm 7.3^{\circ}\right)$. In the present study, the mean correction loss was $8.1^{\circ} \pm 10.2^{\circ}$, and $4 \%(2 / 47)$ of the cases required revision surgery because of instrumentation failure. However, the optimal surgical procedure remains controversial because most studies were retrospective case series with a small sample size. Recently, several reports have demonstrated the efficacy of minimally invasive procedures, including VP and BKP, for OVFs with neurological deficits [23,24]. Nakamae et al. [24] reported that the condition of $84 \%$ of the patients with neurological deficits for OVFs improved after VP. However, VP or BKP are relatively contraindicated and challenging for OVF patients with neurological deficits. The efficacy and safety of VP or BKP in the setting of OVFs with neurological deficits remain unclear.

We identified TP administration as a significant protective factor associated with subsequent VFs. Recent studies have demonstrated preoperative segmental kyphosis, osteoporosis (in case of female patients), and intervertebral cement leakage as risk factors associated with subsequent VFs after BKP or VP [25-27]. In the present study, there were no significant differences in age, sex, fused levels, and pre and postoperative KA between the TP group and non-TP group. TP directly stimulates bone formation and increases bone mineral density (BMD). In a recent literature review, TP reduced the risk of subsequent VFs $[16,17,28]$. Our findings support the therapeutic effect of $\mathrm{TP}$ in OVF patients. In addition, TP increases the BMD and the insertional torque of the pedicle screws and the bone union rate $[29,30]$. These results suggest that TP may have an advantage in terms of instrumentation failure and subsequent VFs after instrumented surgery for OVFs.

In this study, we could not confirm the superiority of $\mathrm{TP}$ in terms of the costs, revision rate, and fusion rate. However, the prevention of subsequent OVF may influence sagittal spinal alignment that decreases the healthrelated quality of life. Multilevel OVFs reportedly increase the risk of chronic pain because of spinal sagittal imbalance [31]. Although the results suggest a significant effect of TP, there are few methodological issues that weaken the study validity. We did not have the BMD data available for most of these patients. In addition, the duration of preoperative TP treatment and the selection criteria of TP treatment were different among the patients. Therefore, there was insufficient information to evaluate the exact therapeutic effect of TP. Furthermore, we did not assess the severity of the vertebral collapse. Finally, the relatively small sample size and the postoperative follow-up period had considerable variation. The postoperative follow-up 
period may not have been long enough to assess the subsequent VFs.

\section{Conclusions}

We present a series investigating the incidence and effect of TP in preventing vertebral body fractures after longinstrumented fusion. This treatment paradigm would be suitable for a prospective trial; however, it represents some evidence that TP may be useful in preventing fractures following long-posterior fusion.

\section{Conflict of Interest}

No potential conflict of interest relevant to this article was reported.

\section{References}

1. Ikeda Y, Sudo A, Yamada T, Uchida A. Mortality after vertebral fractures in a Japanese population. J Orthop Surg (Hong Kong) 2010;18:148-52.

2. Boonen S, Wahl DA, Nauroy L, et al. Balloon kyphoplasty and vertebroplasty in the management of vertebral compression fractures. Osteoporos Int 2011;22:2915-34.

3. Edidin AA, Ong KL, Lau E, Kurtz SM. Morbidity and mortality after vertebral fractures: comparison of vertebral augmentation and nonoperative management in the Medicare population. Spine (Phila Pa 1976) 2015;40:1228-41.

4. Svedbom A, Alvares L, Cooper C, Marsh D, Strom O. Balloon kyphoplasty compared to vertebroplasty and nonsurgical management in patients hospitalised with acute osteoporotic vertebral compression fracture: a UK cost-effectiveness analysis. Osteoporos Int 2013;24:355-67.

5. Taylor RS, Taylor RJ, Fritzell P. Balloon kyphoplasty and vertebroplasty for vertebral compression fractures: a comparative systematic review of efficacy and safety. Spine (Phila Pa 1976) 2006;31:2747-55.

6. Tsujio T, Nakamura $\mathrm{H}$, Terai $\mathrm{H}$, et al. Characteristic radiographic or magnetic resonance images of fresh osteoporotic vertebral fractures predicting potential risk for nonunion: a prospective multicenter study. Spine (Phila Pa 1976) 2011;36:1229-35.

7. Ataka H, Tanno T, Yamazaki M. Posterior instru- mented fusion without neural decompression for incomplete neurological deficits following vertebral collapse in the osteoporotic thoracolumbar spine. Eur Spine J 2009;18:69-76.

8. Kashii M, Yamazaki R, Yamashita T, et al. Surgical treatment for osteoporotic vertebral collapse with neurological deficits: retrospective comparative study of three procedures: anterior surgery versus posterior spinal shorting osteotomy versus posterior spinal fusion using vertebroplasty. Eur Spine J 2013;22:163342.

9. Katsumi K, Hirano T, Watanabe K, et al. Surgical treatment for osteoporotic thoracolumbar vertebral collapse using vertebroplasty with posterior spinal fusion: a prospective multicenter study. Int Orthop 2016;40:2309-15.

10. Nakashima H, Imagama S, Yukawa Y, et al. Comparative study of 2 surgical procedures for osteoporotic delayed vertebral collapse: anterior and posterior combined surgery versus posterior spinal fusion with vertebroplasty. Spine (Phila Pa 1976) 2015;40:E120-6.

11. Okuda S, Oda T, Yamasaki R, Haku T, Maeno T, Iwasaki M. Surgical outcomes of osteoporotic vertebral collapse: a retrospective study of anterior spinal fusion and pedicle subtraction osteotomy. Global Spine J 2012;2:221-6.

12. Sudo H, Ito M, Abumi K, et al. One-stage posterior instrumentation surgery for the treatment of osteoporotic vertebral collapse with neurological deficits. Eur Spine J 2010;19:907-15.

13. Uchida K, Nakajima H, Yayama T, et al. Vertebroplasty-augmented short-segment posterior fixation of osteoporotic vertebral collapse with neurological deficit in the thoracolumbar spine: comparisons with posterior surgery without vertebroplasty and anterior surgery. J Neurosurg Spine 2010;13:612-21.

14. Fribourg D, Tang C, Sra P, Delamarter R, Bae H. Incidence of subsequent vertebral fracture after kyphoplasty. Spine (Phila Pa 1976) 2004;29:2270-6.

15. Mudano AS, Bian J, Cope JU, et al. Vertebroplasty and kyphoplasty are associated with an increased risk of secondary vertebral compression fractures: a population-based cohort study. Osteoporos Int 2009;20:819-26.

16. Tseng YY, Su CH, Lui TN, Yeh YS, Yeh SH. Prospective comparison of the therapeutic effect of teriparatide with that of combined vertebroplasty with 
antiresorptive agents for the treatment of new-onset adjacent vertebral compression fracture after percutaneous vertebroplasty. Osteoporos Int 2012;23:161322.

17. Su CH, Tu PH, Yang TC, Tseng YY. Comparison of the therapeutic effect of teriparatide with that of combined vertebroplasty with antiresorptive agents for the treatment of new-onset adjacent vertebral compression fracture after percutaneous vertebroplasty. J Spinal Disord Tech 2013;26:200-6.

18. Hoshino M, Nakamura $\mathrm{H}$, Terai H, et al. Factors affecting neurological deficits and intractable back pain in patients with insufficient bone union following osteoporotic vertebral fracture. Eur Spine J 2009;18:1279-86.

19. Staples MP, Howe BM, Ringler MD, et al. New vertebral fractures after vertebroplasty: 2-year results from a randomised controlled trial. Arch Osteoporos 2015;10:229.

20. Yang EZ, Xu JG, Huang GZ, et al. Percutaneous vertebroplasty versus conservative treatment in aged patients with acute osteoporotic vertebral compression fractures: a prospective randomized controlled clinical study. Spine (Phila Pa 1976) 2016;41:653-60.

21. Zou J, Mei X, Zhu X, Shi Q, Yang H. The long-term incidence of subsequent vertebral body fracture after vertebral augmentation therapy: a systemic review and meta-analysis. Pain Physician 2012;15:E515-22.

22. Xie W, Jin D, Wan C, et al. The incidence of new vertebral fractures following vertebral augmentation: a meta-analysis of randomized controlled trials. Medicine (Baltimore) 2015;94:e1532.

23. Chen GD, Lu Q, Wang GL, et al. Percutaneous kyphoplasty for Kummell disease with severe spinal canal stenosis. Pain Physician 2015;18:E1021-8.
24. Nakamae T, Fujimoto Y, Yamada K, Takata H, Shimbo T, Tsuchida Y. Percutaneous vertebroplasty for osteoporotic vertebral compression fracture with intravertebral cleft associated with delayed neurologic deficit. Eur Spine J 2013;22:1624-32.

25. Spross C, Aghayev E, Kocher R, Roder C, Forster T, Kuelling FA. Incidence and risk factors for early adjacent vertebral fractures after balloon kyphoplasty for osteoporotic fractures: analysis of the SWISSspine registry. Eur Spine J 2014;23:1332-8.

26. Rho YJ, Choe WJ, Chun YI. Risk factors predicting the new symptomatic vertebral compression fractures after percutaneous vertebroplasty or kyphoplasty. Eur Spine J 2012;21:905-11.

27. Civelek E, Cansever T, Yilmaz C, et al. The retrospective analysis of the effect of balloon kyphoplasty to the adjacent-segment fracture in 171 patients. J Spinal Disord Tech 2014;27:98-104.

28. Bouxsein ML, Chen P, Glass EV, Kallmes DF, Delmas $\mathrm{PD}$, Mitlak BH. Teriparatide and raloxifene reduce the risk of new adjacent vertebral fractures in postmenopausal women with osteoporosis: results from two randomized controlled trials. J Bone Joint Surg Am 2009;91:1329-38.

29. Inoue G, Ueno M, Nakazawa T, et al. Teriparatide increases the insertional torque of pedicle screws during fusion surgery in patients with postmenopausal osteoporosis. J Neurosurg Spine 2014;21:425-31.

30. Ohtori S, Inoue G, Orita S, et al. Teriparatide accelerates lumbar posterolateral fusion in women with postmenopausal osteoporosis: prospective study. Spine (Phila Pa 1976) 2012;37:E1464-8.

31. Zhang YL, Shi LT, Tang PF, Sun ZJ, Wang YH. Correlation analysis of osteoporotic vertebral compression fractures and spinal sagittal imbalance. Orthopade 2017;46:249-55. 